\title{
Cigarettes électroniques (e-cigarettes)
}

Macé M. Schuurmans, Lucrezia Meier-Schatz, Chung-Yol Lee; au nom de la Commission fédérale pour la prévention du tabagisme (CFPT)*

\section{Introduction}

Pour toute discussion sur l'impact de la cigarette électronique, il convient de prendre en compte trois principes:

1. Le principe de réalité: il faut accepter le fait que ces cigarettes sont déjà disponibles et utilisées en Suisse, bien que leurs conséquences sur la santé et la dépendance au tabac ne soient pas encore suffisamment connues.

2. Le principe de prévention: il s'agit de protéger les non-fumeurs, en particulier les enfants et les jeunes, de la consommation d'un produit qui permet d'accéder plus facilement au tabagisme. De plus, il importe d'éviter la banalisation de la fumée, que fumer ne redevienne pas un comportement «normal».

3. Le principe de précaution: les non-fumeurs doivent être protégés (tabagisme passif), car les effets à long terme sur la santé ne sont pas connus.

\section{Etat actuel de la recherche}

a. On entend actuellement par «cigarettes électroniques» une grande variété de produits ayant la même structure: une batterie, un atomiseur (ou vaporisateur), un réservoir ou une cartouche contenant le liquide destiné à être vaporisé (e-liquide), et un embout buccal. Elles sont activées soit par la pression d'un interrupteur, soit par l'aspiration de l'utilisateur au moyen de l'embout, ce qui, par l'intermédiaire d'un capteur, active l'atomiseur ou l'élément chauffant.

b. Les e-liquides contiennent un mélange de propylène glycol, de glycérol et d'eau en proportions variables, ainsi que des arômes. On les trouve avec ou sans nicotine.

c. Actuellement, seuls les liquides ne contenant pas de nicotine sont autorisés à la vente en Suisse. Ceux qui contiennent de la nicotine peuvent être achetés ou commandés sur internet à l'étranger, avec une limite maximale de $150 \mathrm{ml} /$ personne [1].

d. Les utilisateurs de cigarettes électroniques sont principalement des anciens fumeurs ou des fumeurs actuels [2], ainsi que des jeunes et des jeunes adultes [3]. En 2015, le pourcentage des 15 ans et plus qui en ont déjà utilisé au moins une fois (avec ou sans nicotine) était de $14 \%$ en Suisse [3]. La consommation a augmenté chez les plus de 35 ans (entre $24,8$ et $33,0 \%)$, les personnes en formation $(27,4 \%)$ et dans la population de Suisse romande (19,6\%) [3]. Les jeunes et les jeunes adultes sont les utilisateurs les plus fréquents, mais seul un petit pourcentage d'entre eux sont des utilisateurs réguliers (au moins une fois par semaine). On estime que 0,7\% de la population en ferait un usage régulier. Les motivations les plus fréquemment rapportées sont:

- consommer un produit moins toxique que le tabac;

- diminuer la consommation du tabac;

- arrêter de fumer et prévenir la rechute;

- consommer un produit de nicotine moins cher que les cigarettes de tabac;

- utiliser un substitut de tabac dans les endroits où fumer est interdit.

e. Parmi les utilisateurs, on trouve des adolescents, voire des enfants, qui manifestent ainsi leur curiosité, leur besoin d'expérimenter quelque chose de nouveau.

f. Les conséquences négatives pour la santé à court terme sont la sécheresse et les irritations locales (surtout sécheresse naso-pharyngée), les maux de tête, les difficultés respiratoires et la toux [4]. Lorsque les utilisateurs emploient du glycérol non purifié ou modifient les e-liquides, ils risquent, en inhalant de l'huile, de contracter une pneumonie [5]. Des problèmes techniques sont susceptibles de provoquer la fuite de liquide et son absorption par la peau ou les muqueuses, ou d'entraîner une explosion due à la surchauffe, avec les blessures correspondantes $[6,7]$ Un autre risque, en particulier chez les jeunes enfants, est l'intoxication accidentelle à la nicotine par l'ingestion du liquide contenu dans les recharges.

g. Chez les non-fumeurs, la consommation de cigarettes électroniques peut conduire à la dépendance à la nicotine. Le design et les coloris des nombreux modèles proposés ainsi que les divers arômes sucrés des liquides sont particulièrement attirants 
pour les enfants, les adolescents et les jeunes adultes. Il n'est donc pas surprenant que ce groupe d'âge ait envie d'essayer ces cigarettes, s'exposant ainsi à un risque majeur de dépendance à la nicotine.

h. D’autres conséquences à moyen terme sur la santé sont possibles, comme une hypertension, des atteintes pulmonaires et/ou cancéreuses dues aux arômes et autres composés chimiques, tels que le diacyle, le formaldéhyde ou d'autres substances cancérogènes $[5,8,9]$. Les effets de la consommation à moyen et à long terme ne sont pas connus.

i. La cigarette électronique se différencie de la cigarette traditionnelle par le fait qu'elle ne contient pas de tabac et que l'utilisateur n'inhale et ne rejette pas de produits de la combustion de celui-ci. D’après les connaissances scientifiques actuelles, son usage diminuerait, sans l'exclure totalement, le risque de maladies cardio-circulatoires, de cancers et de maladies pulmonaires.

j. A l'heure actuelle, les études (randomisées) prouvant l'efficacité de la cigarette électronique comme moyen de sevrage du tabagisme sont rares. Elles ont été diversement appréciées par les spécialistes, car elles ne sont pas à même de démontrer son effet par rapport aux médicaments de désaccoutumance tabagique enregistrés [10].

k. Le risque de devenir dépendant de la nicotine est élevé avec les cigarettes électroniques qui en contiennent. Ce risque est à surveiller particulièrement chez les jeunes qui ne fument pas de tabac par ailleurs.

1. Le marché de la cigarette électronique avec nicotine pourrait représenter une niche pour l'industrie du tabac, qui s'intéresse de près à ces nouveaux produits, car les ventes de tabac sont en baisse sensible dans de nombreux pays occidentaux.

m. Les avis des experts suisses sont partagés au sujet de la cigarette électronique [11]: certains y voient un instrument potentiellement réducteur de risques, alors que d'autres craignent qu'elle devienne une porte d'entrée vers le tabagisme au niveau individuel et, au niveau social, renormalise le fait de fumer.

n. La CFPT a soutenu la réalisation d'un consensus d'experts (méthode Delphi) et repris la majorité des conclusions de cette étude dans ses recommandations [11]. Elle a également tenu compte, pour l'actualisation de la présente prise de position, des données plus récentes provenant d'autres études ou enquêtes (voir chapitre 4).

o. En l'état actuel des connaissances, les cigarettes électroniques sont moins nocives que les cigarettes contenant du tabac en cas d'usage bref et occasion- nel. En raison du manque de données relatives à leurs effets à moyen et à long terme, il n'est pas encore possible d'estimer avec exactitude dans quelle mesure la substitution totale des cigarettes ordinaires par leurs pendants électroniques entraînerait une réduction relative des risques. Une analyse de ce type réalisée en Grande-Bretagne est critiquée par les experts [12].

\section{Recommandations}

1. Les cigarettes électroniques doivent faire l'objet d'une régulation spécifique: normes de qualité pour les spécifications des appareils et pour le contenu des cartouches (liquides), liste restrictive de composants autorisés dans ces liquides et limite maximale de concentration pour la nicotine. La composition doit figurer sur l'emballage, de même que des mises en garde sur les risques liés à la consommation.

2. La loi fédérale sur la protection contre le tabagisme passif et les législations cantonales complémentaires doivent également s'appliquer à la cigarette électronique.

3. Toutes formes de promotion et de publicité doivent être interdites, parce que les groupes cibles vulnérables (enfants et jeunes) sont toujours visés et parce que les résultats des études montrent que la consommation de cigarettes électroniques augmente chez les jeunes le risque de tabagisme par la suite.

4. En accord avec les recommandations de l'OMS, les autorités sanitaires ne doivent pas recommander la cigarette électronique pour le sevrage du tabagisme [13], en raison du manque de données sur la sécurité et l'efficacité à long terme. Il convient de recommander les traitements ayant déjà fait la preuve de leur efficacité (produits de substitution, bupropion, varénicline, ligne stop-tabac, conseils médicaux et non médicaux, etc.).

5. La vente des cigarettes électroniques contenant de la nicotine doit être réservée aux adultes.

6. Une taxe doit être prélevée sur les cigarettes électroniques, d'un montant équivalent à celle prélevée sur les cigarettes ordinaires. Le produit de cette taxe doit servir à financer la prévention du tabagisme et la recherche sur ce type de cigarettes, notamment sur ses effets à long terme sur la santé, son efficacité comme moyen de sevrage du tabac, ses conséquences psychologiques et sociales, ainsi que sur la consommation simultanée de produits du tabac et de cigarettes électroniques.

7. Des analyses régulières des e-liquides les plus vendus sont indispensables. A cet effet, une coordination entre cantons serait utile. 


\section{Références}

1 Charrière R. Informationsschreiben Nr. 146: Elektrische Zigaretten, elektronische Zigaretten, E-Zigarette. 2010. BAG, Bern.

2 Etter JF, Bullen C. Electronic cigarette: users profile, utilization, satisfaction and perceived efficacy. Addiction, 2011;106(11):2017-28.

3 Kuendig H, Notari L, Gmel G. Die E-Zigarette in der Schweiz im Jahr 2015 - Auswertung der Daten des Suchtmonitorings Schweiz. 2016. Sucht Schweiz, Lausanne.

4 Caponnetto P, et al. Efficiency and Safety of an eLectronic cigAreTte (ECLAT) as tobacco cigarettes substitute: a prospective 12-month randomized control design study. PLoSONE, 2013;8(6):e66317.

5 Hua M, Talbot P. Potential health effects of electronic cigarettes: A systematic review of case reports. Prev Med Rep. 2016 Jun 10, 4:169-78.

6 Maina G, Castagnoli C, Passini V, Crosera M, Adami G, Mauro M, Filon FL. Transdermal nicotine absorption handling e-cigarette refill liquids. Regul Toxicol Pharmacol. 2016 Feb;74:31-3.

7 Nicoll KJ, Rose AM, Khan MA, Quaba O, Lowrie AG. Thigh burns from exploding e-cigarette lithium ion batteries: First case series. Burns. 2016 Jun;42(4):e42-6.
8 Schuurmans MM. Elektronische Zigaretten und kardiovaskuläres Risiko. Herz+Gefäss 2016;1:6-9.

9 Farsalinos KE, Kistler KA, Gillman G, Voudris V. Evaluation of electronic cigarette liquids and aerosol for the presence of selected inhalation toxins. Nicotine Tob Res. 2015 Feb;17(2):168-74.

10 Kaelin R, Barben J, Schuurmans MM. Elektronische Zigaretten, E-Shishas und "Heat but not burn devices». Swiss Med Forum. 2017:17(5) (in print)

11 Blaser J, Cornuz J. Experts' consensus on use of electronic cigarettes: a Delphi survey from Switzerland. BMJ Open. 2015 Apr 15; 5(4):e007197.

12 E-cigarettes: Public Health England's evidence-based confusion. Lancet. 2015 Aug 29;386(9996):829.

13 WHO: WHO study group on tobacco product regulation (TobReg): report on the scientific basis of tobacco product regulation: third report of a WHO study group, WHO technical report series no. 955, 2009. http://www.who.int/tobacco/global_interaction/tobreg 